\title{
Improve Reliability and Load Balancing of using ACO Mechanism in Ad hoc Network
}

\author{
Amit Chandra Pathak \\ M.Tech Scholar \\ V.N.S. Institute of Technology \\ Bhopal, (M.P.), India
}

\author{
Shashikant Pandey \\ Assistant Professor \\ V.N.S. Institute of Technology \\ Bhopal, (M.P.), India
}

\begin{abstract}
Ad hoc network consist of autonomous self-organized nodes. Nodes use a wireless medium for communication. Thus two nodes can communicate directly if and only if they are within each other's transmission radius. In Ad hoc networks, routing not only has to be fast and efficient, but also adaptive to the changes in the network topology; otherwise, the performance may be severely degraded. In a routing task, a message is sent from a source to a destination node in a given network. Two nodes normally communicate via other nodes in a multi-hop fashion. In this paper we analyze an ACO (Ant Colony Optimization) based routing algorithm with AOMDV protocol for load balancing to route packets through shorter and feasible routes. Each ant while moving towards destination collects information about address of each visited node of the followed path means the pheromones values. During backward travel, local network traffic model and routing table is modified by ant based on the goodness of the followed path. The performance of ACO with AOMDV has provides the possibility of light load traffic routes that is not possible to find in normal AOMDV routing protocol and provides the efficient load balancing on the basis of high and low pheromones value. Simulation results shows that the proposed ACO based routing protocol gives performance improvement over other routing protocol by continuously checking for better paths in the network with less overhead.
\end{abstract}

\section{Index Terms}

Routing, Ad hoc network, AOMDV, ACO, Load balancing

\section{INTRODUCTION}

In mobile ad hoc network (MANET), the nodes work together in a distributed fashion to enable routing among them [1]. Because of the lack of centralized control, routing becomes a central issue and a major challenge as the network topology is constantly changing. It is a collection of mobile nodes that are dynamically and arbitrarily located in such a manner that the interconnections between nodes are capable of changing on a continual basis. Each node can act as a receiver, transmitter or router [2]. The main problem of the ad-hoc network is mobility of the nodes resulting in fast variations of their availability. At one time the node is in range and while at other that node is out of the range.

One interesting research area in MANET is routing [3]. In MANETs the transmission range of nodes is limited so the nodes in MANETs act as both hosts and routers. The routing protocol for MANETs needs to be flexible enough to adapt to the continuously changing network topologies, and to support bandwidth management

\subsection{Need for Ad Hoc Networks}

i Setting up of fixed access points and backbone infrastructure is not always viable ii Infrastructure may not be present in a disaster area or war zone

iii Infrastructure may not be practical for short-range radios Bluetooth (range $\sim 10 \mathrm{~m}$ )

iv Ad hoc networks are not need backbone infrastructure support

v Are easy to deploy

vi Useful when infrastructure is absent, destroyed or impractical.

The AOMDV Protocol (Ad Hoc on-demand Multipath Distance Vector Routing) [4] is basically multipath extensions on top of AODV. The route discovery process has been modified to enable multiple paths. They stress on link disjoint ness of multiple paths such that the paths may share nodes but no edges. Also the loop freedom property of paths is guaranteed by using sequence numbers of nodes. After mentioning link disjoint ness with a high importance, it is interesting that the authors prefer to use one path at a time rather than simultaneous usage of multiple paths. Their reason to choose single path at a time is the requirement of addressing issues, splitting traffic along each path and packet reordering at the destination. And as a different aspect of AOMDV than AODV, the usage of periodic HELLO messages to detect stale paths can be mentioned.

The proposed scheme has balanced the load in network by using the ACO scheme with multipath routing to balance the load in network properly and the path has selected on the basis of traffic load in network.

\section{ACO IN AD HOC NETWORK}

Recently, nature's self-organizing such as ant colony optimization algorithms [5] are proved to be efficient in developing routing algorithm for Ad hoc network. Ant Colony Optimization (ACO) is based on the capability of real ant colonies of finding the shortest path from nest to food source [5]. Ant uses pheromone to communicate with other ants. Pheromone is a chemical deposited on ground by the ants while walking and it affect their moving decisions based on the intensity. The ants are reaching to goal use following steps

\section{Initially ants choose path randomly}

2. Ant choosing shorter path will reach first

3. Pheromone deposited more quickly on shorter path, number of ants keep on increasing on sorter path.

These ants deposit pheromone on the ground in order to mark some favorable path to reach the food source that should be followed by other member of the colony. Ant colony optimization exploits similar mechanism for solving optimization problems. 


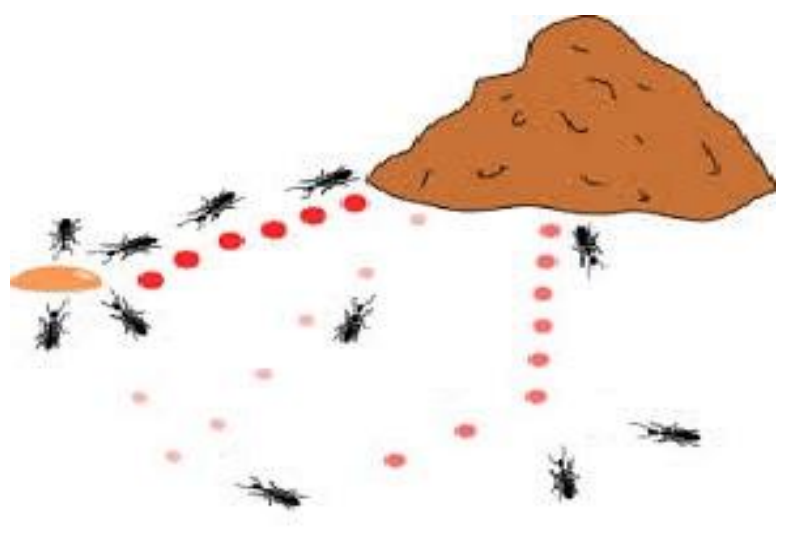

Fig. 1. Ant Colony Optimization

An ant going from source node source $(\mathrm{S})$ to destination node destination (D) collects information about the quality of the path and uses this on its way back from $\mathrm{D}$ to $\mathrm{S}$ to update the routing information at the intermediate nodes shown in Figure 1. In this figure the red color mentioned the higher pheromones value and the rest of the path has low pheromones value. It means that

In Ant Colony Optimization routing technique has provides better paths among all available paths have higher pheromone concentration. Thus, a path consisting of a series of links with highest pheromone values is always the favorable path between any source destination pair. The protocol also calculates next best path having lower pheromone value which would be helpful in selecting next best alternative path in case of link failures, without additional overheads. The basic idea behind ACO algorithms for routing [5] is the acquisition of routing information through sampling of paths using agents, which are called ants. In this paper ACO based network routing algorithm is analyzed inspired by the foraging behavior of the ants, to route packets through shorter and feasible routes. The remainder of this paper is organized as follows.

In section 2 the Ant colony Optimization Technique is described and the related work is mentioned in section 3. The proposed scheme is described in section 4. In section 5 the proposed scheme as mentioned and in section 5 measures performance of proposed scheme through simulation results. Finally conclude this work in section 7

\section{LITERATURE SURVEY}

Bibhash Roy et. al. [7] proposed Ant Colony based Routing for Mobile Ad-Hoc Networks towards Improved Quality of Services. A brand new Quos algorithmic rule for mobile ad hoc network has been planned. The projected algorithmic rule combines the thought of ant Colony optimisation (ACO) with Optimized Link State Routing (OLSR) protocol to spot multiple stable methods between supply and destination nodes. The challenges reside in ad hoc networks is to seek out a path between the communication finish points satisfying user's QoS demand which require to be maintain consistency. The rule consists of each reactive and proactive element. In a very reactive path setup section, a choice of multiple methods selection will be accustomed build the link between the source and destination during an information session.

S. Soundararajan et al. [8] describes about Ant Based Multi-path Routing for Load Balancing and Congestion Control in MANETs. A lot of message overheads are caused attributable to enlarged flooding. Packets are dropped by midway nodes because of frequent link failures. What is more the general turnout and therefore the packet delivery quantitative relation is reduced in high quality situations. so as to beat the problems an efficient multi path routing protocol ABMRLBCC (Ant based mostly Multi-path Routing for Load levelling and Congestion Control) supported ant Colony improvement is projected. Once the network size and therefore the speed of the mobile nodes are increased, it shows higher performance in terms of packet delivery quantitative relation with reduced delay and overhead.

M. Ahmed et al. [9] propose two ways to enhance the Ad-Hoc On-Demand Distance-Vector (AODV) protocol [3]. The main goal within the design of the protocol was to reduce the routing overhead, buffer overflow, end-to-end delay and increases the performance. A multi-path routing protocol is projected which relies on AODV and ant Colony optimization (ACO). This protocol is refereed to Multi-Route AODV ant routing (MRAA). Also we have a tendency to propose a load balancing technique that uses all discovered ways at the same time for transmittal knowledge. During this technique, information packets are balanced over discovered ways and energy consumption is distributed across many nodes through network.

Anuj K. Gupta et al. in [10] through this paper provide a platform for researchers worldwide to get an overview of the existing ACO based routing protocols. To know about their performance against traditional ad hoc routing protocols. This would rather help them consider appropriate protocol for their research work. The authors have tried their best to present a comparative study of various proposed ad hoc routing protocols based on ant colony optimization techniques.

Pengxiu Zhang et al. [11] proposed a Ant-Colony-based Routing Protocol (ACRP) for DTMNs. The pheromone on the nodes indicates the delivery probability and is used to decide which node should carry the message. Forward ants and backward ants are used to establish the pheromone trail. And the data ants make forwarding decision according to the probability function composing both the pheromone and the heuristic information. Also, proper forwarding actions are chosen regarding the pheromone of the next hop.

G. di Caro et al. in [12] proposed is a new algorithm for routing in mobile ad hoc networks. It consists of both reactive and proactive components. In a reactive path setup phase, multiple paths are set up between the source and destination of a data session, using hop count and queuing delay to calculate pheromone, and during the course of the communication session, ants proactively test existing paths and explore new ones.

M. Belkadi et al. in [13] proposed a new QoS routing protocol combined with the flow control mechanism. This proposed routing solution is modeled by ant systems. The proposed routing protocol uses a new metric to find the route with higher transmission rate, less latency and better stability.

Shahab Kamali. et.al [14] implemented a new ant colony based routing algorithm that uses the information about the location of nodes.

\section{PROPOSED SCHEME}

The proposed ACO with AOMDV scheme based routing algorithm exploits a dual-mode approached. The network topology changes were constantly monitored. When the rate of topology changed got too high for ants to converge efficiently, a node switched in network frequently. The proposed approach were significantly improved the performance of the network by quickly adapting to the dynamics of the environment. Routing protocols use metrics to evaluate what path will be the best for a packet to travel. The process of path selection and directing packets delivery in network between sender nodes to the 
receiver nodes in routing based on the pheromones value. The AOMDV are capable to balancing load but only by providing the alternative path but ACO with AOMDV are also balance the load and provides the most of the alternative routes and the shortest route is selected on the basis of higher pheromones value. It the current path is congested then in that case the next higher pheromone value path is selected as alternative route.

The path selections is depend on the traffic of network it means if traffic is high then select the higher pheromone path and in low traffic condition select low pheromones value path.

The proposed algorithm are clear the actual routing procedure.

1. Set Routing Protocol AOMDV

2. Set Load Analysis Protocol ACO

3. Set R_Packets is a Routing Packets

4. Set R_ACK is a Route Acknowledgement

5. Broadcast Route_Request (S, R, Route_Request)

6. If (Node in Radio Range \&\& Node!=R)

7. \{

8. Receive R_Packets

9. Create pheromones table of nodes;

10. \}

11. If $($ Node $==R) \& \&($ Path $\geq 1)$

12. \{

13. Receive R_Packets

14. Create Reverse Path

15. Send R_ACK \&\& Pheromones value of each node.

16. $\}$

17. Sender receive R_ACK \&\& pheromones value of each node.

18. Assign priority order base path using high pheromone value.

19. Send maximum data via higher pheromones value path.

20. Send minimum data via low pheromones value base path

21. Stop.

\section{SIMULATION ENVIRONMENT}

Network simulator 2 (NS2) is the result of an on-going effort of research and development that is administrated by researchers at Berkeley [14]. It is a discrete event simulator targeted at networking research. It provides substantial support for simulation of TCP and UDP Protocols, multipath protocol.

\subsection{Simulation Parameter}

Let's get Evaluation Parameter like Number of nodes, Dimension, Routing protocol, transport layer protocol, application layer data and maximum speed of mobile nodes etc. According to below table 1.1 we simulate our network.
TABLE I. Simulation Parameters

\begin{tabular}{|l|l|}
\hline Number of nodes & 50 \\
\hline Dimension of simulated area & $800 \times 600$ \\
\hline Radio Range (meters) & 250 \\
\hline Routing Protocol & AOMDV \\
\hline Simulation time (seconds) & 100 \\
\hline Transport Layer & TCP ,UDP \\
\hline Traffic type & FTP, CBR \\
\hline Packet size (bytes) & 1000 \\
\hline Number of traffic connections & 10 \\
\hline Nodes Speed (m/s) & Random \\
\hline
\end{tabular}

\subsection{Performance Matrices}

We simulate our result on the basis of following performance parameter are as follows.

\section{Packet Delivery Ratio}

The ratio between the number of packets originated by the sources and the number of packets received by the sink at the final destination.

\section{Routing Load}

The total number of routing packets transmitted during the simulation. For packets sent over multiple hops, each transmission of the packet or each hop counts.

\section{Packet Dropped}

The routers might fail to deliver or drop some packets or data if they arrive when load in network are more. Some, none, or all the packets or data might be dropped, depending on the state of the network, and it is impossible to determine what will happen in advance.

\section{SIMULATION RESULTS}

This section is mentioned the simulation results that has evaluated after applying proposed algorithm. These results are showing the performance of normal AOMDV routing protocol and ACO with AOMDV routing protocol.

\subsection{Packet Delivery Ratio analysis}

The Packet Delivery Ratio (PDR) ia the percentage ratio of number of packets successfully received at destination. The normal AODV routing protocol has not the capability to provides the more than one path in between sender and receiver in network but ANT colony optimization technique having a capability to improves the routing capability of normal AOMDV routing protocol by proving the number of alternatives in network for deliver data in between sender and receiver through intermediate nodes. In this graph the performance of normal AOMDV (PDR-W-ANT) means the routing with AOMDV protocl without ANT technique and AODV with ACO (Ant Colony Optimization) (PDR-ANT) are evaluate on the basis of PDR matrices. Here we clearly visualized that the PDR in case of normal AODV routing is about $90 \%$ but in case of proposed ANT based AODV routing is about $98 \%$, it means in term of PDF about $8 \%$ more improvement in proposed scheme. 


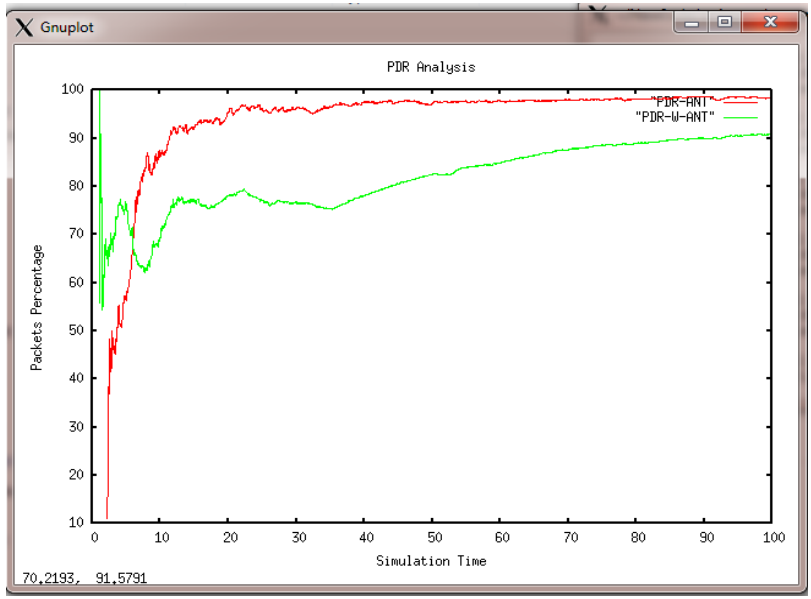

Fig. 2. PDR Analysis

\subsection{Routing Load analysis}

Routing load is measured on the basis of number of routing packets or connection established packets are delivering in network. The requirement of routing packet is to find the destination and after that the data delivery is started and if the link is break in network due to mobile nature of network then again is needed to first established connection in network and successfully deliver data in network. It means that also the more number of routing packets delivery are degrades the network performance and enhance the routing load in network. In this graph the performance of routing protocols are measured on the basis of routing load and we identified that the performance of proposed ANT with AOMDV are less as compare to normal AODV routing. Here in case of normal AOMDV routing about 2500 routing packets are delivering in network but in case of proposed ANT based routing scheme about 1300 routing packets re deliver in network at simulation time 99 seconds it means that only one second no routing packet is deliver but is deliver because in proposed scheme that provides the multiple paths by that the routing load minimizes.

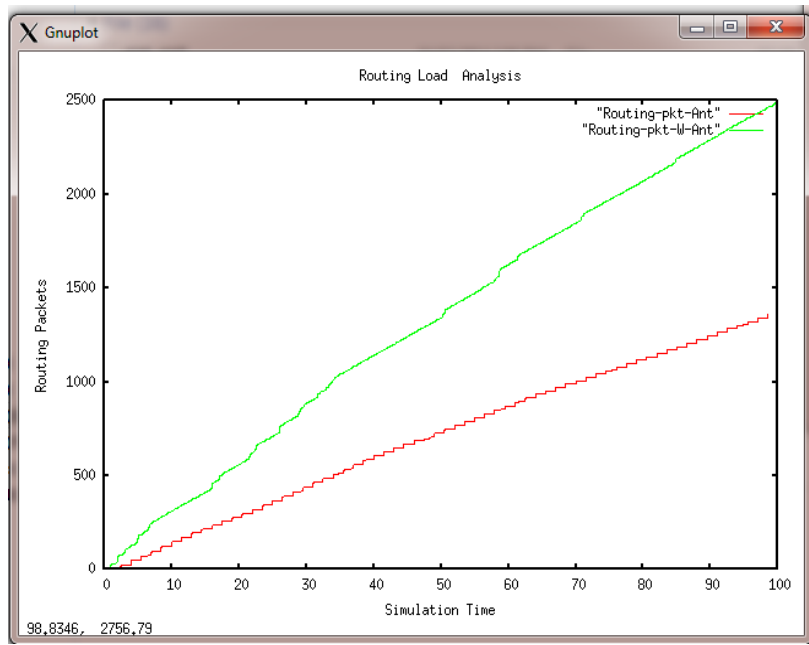

Fig. 3. Routing Load Analysis

\subsection{TCP Packets Analysis without ANT}

Transmission control protocol (TCP) is the connection oriented protocol that provides the reliable data deliver in network. The connection oriented mechanism is based on the ACK (Acknowledgement) based scheme in which the fist connection is established with receiver ten the data delivery is started and if the ACK of data is received then the next delivery is started. This graph represents the TCP congestion window in case of normal AODV routing protocol. Here we observe that the highest size of congestion window is about 58 of TCP connection TCP2-W_Ant and the performance of rest of the connections is much lower.

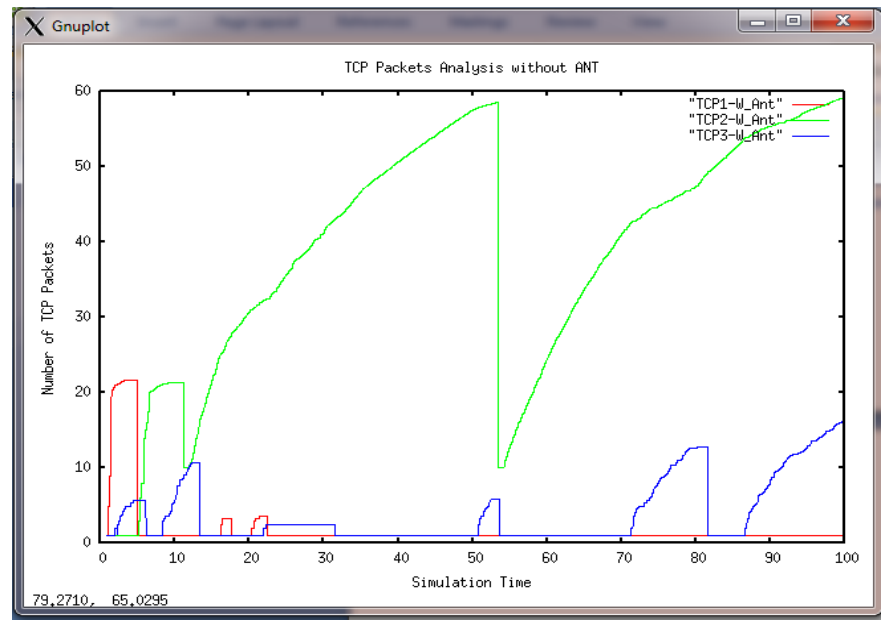

Fig. 4. Normal TCP Packet Analysis

\subsection{TCP Packet Analysis with ANT}

The TCP connection is reliable and their reliability is deliberate on the basis of network conditions it means that if the network traffic is more than in that case the possibility of packets dropping is more but this packet dropping is controlled by TCP protocol by ACK scheme. This graph shows the performance of AOMDV routing protocol with Ant colony scheme. The AOMDV protocol are not capable alone to handle the possibility of heavy traffic because of presence of multiple paths selected on the basis of pheromones value but of the It means that the Ant based AOMDV has provides the better performance as normal AODV.

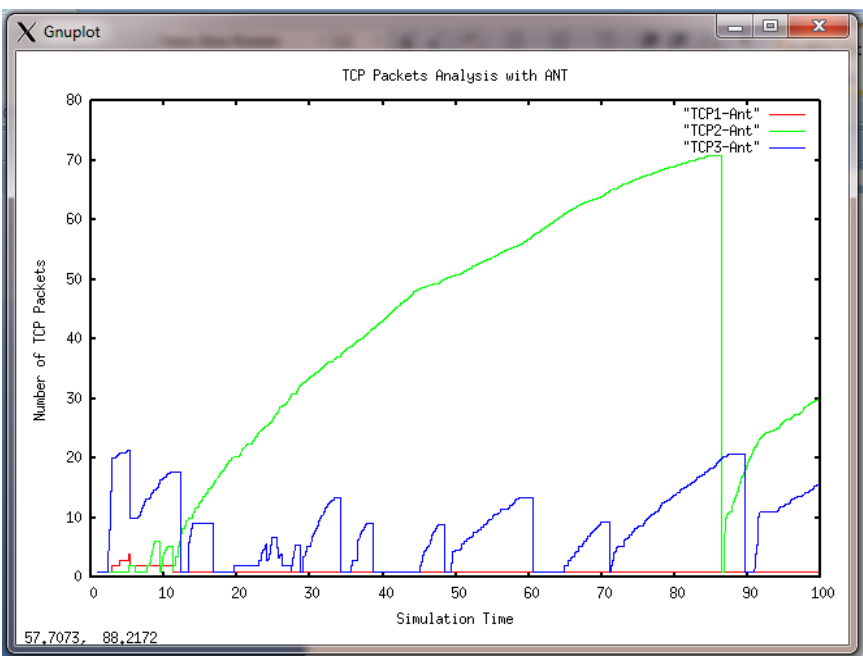

Fig. 5. TCP Packet Analysis with ANT

\subsection{UDP Packets Receiving Analysis}

User datagram Protocol (UDP) are connection less unreliable protocol for data delivery in network. This protocol performance are completely depends on the network conditions. it means the network load is more than the possibility of success data delivery is not sure because of no ACK of data deliver is received by sender through receiver. In this graph the performance of UDP packet receiving is deliberate in case of normal AOMDV routing and in case of AOMDV routing with ACO. The performance of AOMDV with ACO packet receiving is much better and about 2300 packets are received and in case 
of AOMDV routing the packet receiving is about 1800. It means the ACO has improves the performance of UDP protocol.

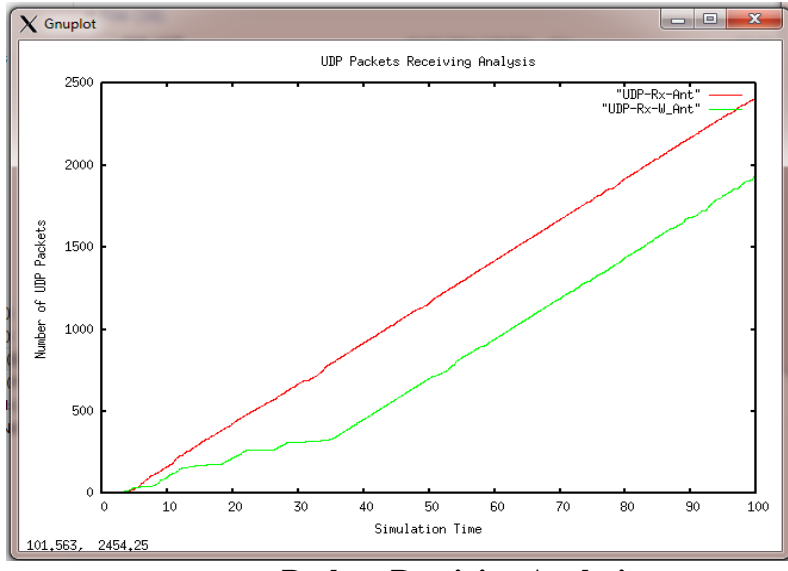

Fig. 6. Packets Receiving Analysis

\subsection{UDP Packets Lost Analysis}

The packet loss is the major issue in network and it is more sensitive in case if the protocol is less reliable for communication. This graph represents the UDP packet loss analysis in case of normal AOMDV routing and in case of AOMDV with ACO. Here we clearly observe that in case of normal AOMDV routing about 510 packets are loss in network but in case of proposed AOMDV routing with ACO only 620 packets are received in network. The ACO based routing is efficiently handle the load as compare to load balancing of AOMDV routing protocol.

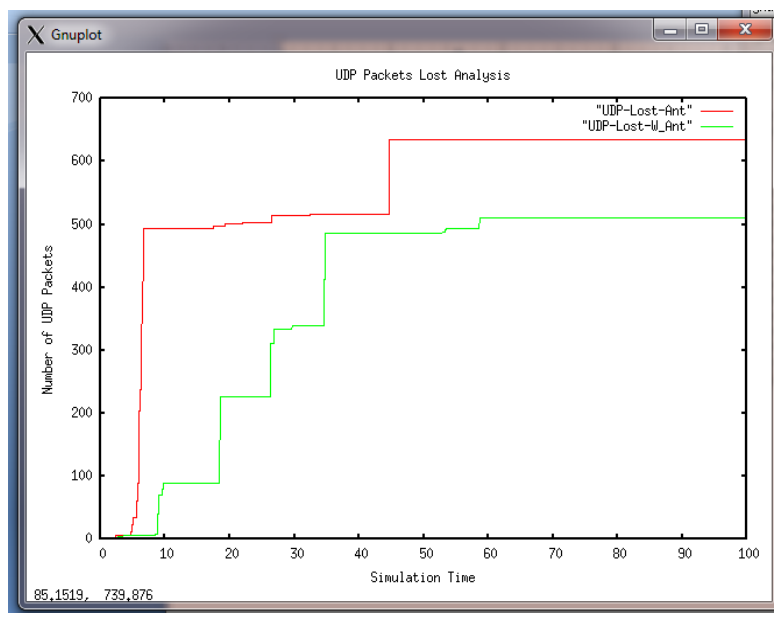

Fig. 7. Packets Lost Analysis.

\section{CONCLUSION}

MANETs are highly dynamic and distributed in nature. The researches done have shown that nature inspired routing protocols can remove at least one or several problems in the area such as battery life, scalability, maintainability, survivability, adaptability and so on. In general, we note that it is very difficult to design routing protocols which are scalable under extreme traffic conditions, but incorporating congestion awareness complicates the problem by incurring overheads in an already heavy loaded network. In ACO technique Ants leave pheromone trails at nodes or edges which increase the likelihood of other ants to follow these trails. In proposed scheme routing paths are then selected on the basis of traffic density, using this to balance the load in network traffic. The trade-off between the amount of overhead expended in finding congestion-free paths and data delivery is very delicate at high traffic loads. However, we see that proposed scheme is able to achieve this balance and perform better than AOMDV in higher traffic load conditions and improving the routing capability in network.

\section{REFERENCES}

[1] C.-K. Toh. Ad hoc mobile wireless networks: protocols and systems. Prentice Hall, 2002. ISBN: 0-13-007817-4.

[2] C. E. Perkins, “Ad hoc Networking”, Pearson Publication.

[3] K. A. Gupta, Harsh Sadawarti, K. A. Verma, "Performance analysis of AODV, DSR and TORA Routing Protocols," International Journal of Engineering and hnology (IJET), ISSN: 1793-8236, Article No. 125, Vol.2 No. 2, April 2010.

[4] M. K. Marina and S. R. Das. On-demand multipath distance vector routing in ad hoc networks. Proceedings IEEE ICNP, pages $14-23,2001$.

[5] Marco D Arigo and Thoma Stutzle, "Ant Colony Optimization", MIT Press Landon, England.

[6] Anubhuti Verma"Ad hoc Network Routing Optimization and Performance Analysis of ACO Based Routing Protocol" International Journal of Advanced Research in Computer Engineering \& Technology Volume 1, Issue 1, March 2012

[7] Bibhash Roy, Suman Banik, Parthi Dey, Sugata Sanyal, Nabendu Chaki, " Ant Colony based Routing for Mobile Ad-Hoc Networks towards", Volume No.3, January 01, 2012.

[8] S. Soundararajan, R. S. Bhuvaneswaran, "Ant Based Multipath Routing for Load Balancing and Congestion Control in MANETs", Journal of Information \& Computational Science, 2012

[9] Ahmed M. Abd Elmoniem, Hosny M. Ibrahim, Marghny H. Mohamed, and Abdel-Rahman Hedar,"Ant Colony and Load Balancing Optimizations for AODV Routing Protocol", Ash din Publishing International Journal of Sensor Networks and Data Communications Vol. 1, 2012.

[10] Anuj K. Gupta, Harsh Sadawarti, and Anil K. Verma "MANET Routing Protocols Based on Ant Colony Optimization" International Journal of Modeling and Optimization, Vol. 2, No. 1, February 2012.

[11] Pengxiu Zhang, Haiquan Wang, Chunhe Xia, Liangshuang Lv, Xiaodong Liu, "ACRP: Ant-Colony-based Routing Protocol for DTMNs", IEEE International Conference on Educational and Information Technology (ICEIT 2010), pp. 272-276, 2010

[12] G. di Caro, F. Ducatelle, and L. M. Gambardella, "AntHocNet: an ant-based hybrid routing algorithm for mobile ad hoc networks," Lecture Notes in Computer Science, pp. 461-470 2004

[13] M. Belkadi, M. Lalam, A. M'zoughi, N. Tamani1, M. Daoui and R. Aoudjit, "Intelligent Routing and Flow Control in MANETS," Journal of Computing and Information Technology - CIT, pp.233-243, March 18, 2010.

[14] Shahab Kamali and Jaroslav Opatrny, "A Position Based Ant Colony Routing Algorithm for Mobile Ad-hoc Networks," Journal of Networks, Vol. 3, No. 4, April 2008.

[15] Marc Gries, Tutorial for Network Simulator-ns, http:// www.isi. edu/nsnam/ns/tutorial. 